This paper was originally accepted for publication by the Pacific Journal in 1993 . Unfortunately it got lost and was therefore not published at that time. This was discovered in 2004. Since Professor Kempf had passed away it took some time to recover the manuscript. We are deeply indebted to Enrico Arbarello, David Mumford, Linda Ness, Christopher Sogge, and Christina Stanger for helping us locate this paper - Managing Editor. 
Pacific

Journal of

Mathematics

A THETA DIVISOR CONTAINING AN ABELIAN SUBVARIETY

George KempF 


\title{
A THETA DIVISOR CONTAINING AN ABELIAN SUBVARIETY
}

\author{
George KempF
}

\begin{abstract}
We construct a Jacobian of dimension three whose theta divisor contains an elliptic curve. We work over an algebraically closed field of characteristic zero.
\end{abstract}

Let $E$ be an elliptic curve and $F$ a principally polarized abelian variety of dimension 3. Let $\mathcal{L}$ and $\mathcal{N}$ be their principal polarizations.

Lemma 1. There exist $E^{\prime}$ and $F^{\prime}$ such that we have isogenies $\psi_{E}: E^{\prime} \rightarrow E$ and $\psi_{F}: F^{\prime} \rightarrow F$ of degree two.

Proof. Let $F^{\prime}=\left(F^{`} / \mathbf{Z} / 2 \mathbf{Z}\right)^{`}$ and the same with $E$.

Let $\mathcal{L}^{\prime}=\psi_{E}^{*} \mathcal{L}$ and $\mathcal{N}^{\prime}=\psi_{F}^{*} \mathcal{N}$. Then $\varphi_{\mathcal{L}^{\prime}}: E^{\prime} \rightarrow\left(E^{\prime}\right)^{`}$ and $\varphi_{\mathcal{N}^{\prime}}: F^{\prime} \rightarrow$ $\left(F^{\prime}\right)^{`}$ have degree four. Let $H_{\mathcal{L}^{\prime}}$ and $H_{\mathcal{N}^{\prime}}$ be their kernels. Then we have theta groups

$$
1 \rightarrow \mathbf{G}_{m} \rightarrow G_{\mathcal{L}^{\prime}} \rightarrow H_{\mathcal{L}^{\prime}} \rightarrow 0
$$

and

$$
1 \rightarrow \mathbf{G}_{m} \rightarrow G_{\mathcal{N}^{\prime}} \rightarrow H_{\mathcal{N}^{\prime}} \rightarrow 0 .
$$

By Mumford theory we have two torsion elements $\alpha_{\mathcal{L}}$ and $\beta_{\mathcal{L}}$ of $G_{\mathcal{L}^{\prime}}$ such that $\alpha_{\mathcal{L}} \cdot \beta_{\mathcal{L}}=(-1) \beta_{\mathcal{L}} \cdot \alpha_{\mathcal{L}}$ and the same with $\mathcal{N}$. Here the images of $\alpha_{\mathcal{L}}$ and $\beta_{\mathcal{L}}$ generate $H_{\mathcal{L}^{\prime}}$. Consider $\mathcal{M}=\pi_{E^{\prime}}^{*} \mathcal{L}^{\prime} \otimes \pi_{F^{\prime}}^{*} \mathcal{N}^{\prime}$. Then $H_{\mathcal{M}}=H_{\mathcal{L}^{\prime}} \times H_{\mathcal{N}^{\prime}}$.

Lemma 2. We have an inclusion $K=(\mathbf{Z} / 2 \mathbf{Z})^{2} \subset G_{\mathcal{M}}$.

Proof. $\alpha_{\mathcal{L}} \otimes \alpha_{\mathcal{N}}$ and $\beta_{\mathcal{L}} \otimes \beta_{\mathcal{N}}$ generate the group.

Let $X=E^{\prime} \otimes F^{\prime} / \operatorname{Im} K$ and let $R$ be the quotient of $\mathcal{M}$ by $(\mathbf{Z} / 2 \mathbf{Z})^{2}$. Then $R$ gives a principal polarization on $X$. Let $\gamma$ be a nonzero section of $X$. Let $\theta$ be the zeroes of $\gamma$.

Lemma 3. $\theta$ contains some translate of $\operatorname{Im} E^{\prime}$.

Proof. $\gamma$ corresponds to a section of $\mathcal{M}$ that is invariant under $K$. Let $\tau$ and $\mu$ be nonzero sections of $\mathcal{L}^{\prime}$ and $\mathcal{N}^{\prime}$ invariant under $\alpha_{\mathcal{L}}$ and $\alpha_{\mathcal{N}}$. Let $\tau^{\prime}=\beta_{\mathcal{L}}(\tau)$ and $\mu^{\prime}=\beta_{\mathcal{N}}(\mu)$. Then $\tau^{\prime}$ and $\mu^{\prime}$ are anti-invariant under $\alpha_{\mathcal{L}}$ and $\alpha_{\mathcal{N}}$. Consider the section $\eta=\tau \otimes \mu+\tau^{\prime} \otimes \mu^{\prime} \neq 0$ of $\mathcal{M}$. Then $\eta$ is invariant under $(\mathbf{Z} / 2 \mathbf{Z})^{2}$. Then the inverse image of $\theta$ is the zeroes $\eta \supset E \times\left(\mu=\mu^{\prime}=0\right)$, where the second set is nonempty as $\mathcal{N}^{2}$ is ample. 
Assume that $F$ contains no elliptic curve.

Lemma 4. $\left(\mu=\mu^{\prime}=0\right)$ is a finite set.

Proof. Let $D$ be the largest divisor in the intersection. Then $D$ is invariant under the group $P$ generated by the image of $\alpha_{\mathcal{N}}$ and $\beta_{\mathcal{N}}$. Then $D$ comes from an effective divisor $D^{\prime}$ on $F^{\prime} / P$ where $\# P=4$. So $\frac{\left(D^{2}\right)}{4}=4 \frac{\left(D^{\prime}\right)^{2}}{2}$ and $\frac{\left(D^{2}\right)}{2} \leq \frac{(\mu=0)^{2}}{2}=2$. So by $[\mathbf{1}], D^{\prime}$ comes from a divisor on a quotient of $F^{\prime} / D$ which is a point. So $D^{\prime}$ is empty.

Lemma 5. $(X, \theta)$ is a Jacobian.

Proof. We need to see that $\theta$ is irreducible. If $\theta$ is reducible, we have $X=$ $E \oplus R$ by [1], where $\theta$ is the sum of divisors depending on the factors. Thus $\theta \supset E \times x$ for a curve $x$. But this contradicts Lemma 4 .

\section{References}

[1] G. Kempf, Complex Abelian Varieties and Theta Functions, Universitext, Springer Verlag, Berlin, 1991, MR 1109495 (92h:14028), Zbl 0752.14040.

[2] D. Mumford, Varieties defined by quadratic equations, Questions on Algebraic Varieties (C.I.M.E., III Ciclo, Varenna, 1969), 29-100, Edizioni Cremonese, Rome, 1970, MR 0282975 (44 \#209), Zbl 0198.25801.

Received May 20, 1993

Department of Mathematics

JOHNS HOPKINS UNIVERSITY

BALTIMORE MD 21218 